\title{
DIAGNOSTICS OF PLASMA JETS IN THE SOLAR CORONA
}

\section{S.A. Anfinogentov \\ Institute of Solar-Terrestrial Physics SB RAS, Irkutsk, Russia, anfinogentov@iszf.irk.ru}

\section{T.I. Kaltman}

Special Astrophysical Observatory RAS,

St.Petersburg,Russia, arles@mail.ru

\section{A.G. Stupishin}

St Petersburg University,

St.Petersburg, Russia, agstup@yandex.ru

\section{V.M. Nakariakov}

Special Astrophysical Observatory RAS,

St.Petersburg, Russia,v.nakariakov@warwick.ac.uk

Centre for Fusion, Space and Astrophysics,

University of Warwick, UK, v.nakariakov@warwick.ac.uk

\author{
M.A. Loukitcheva \\ Special Astrophysical Observatory RAS, \\ St.Petersburg, Russia,m.lukicheva@spbu.ru \\ St Petersburg University, \\ St. Petersburg, Russia, m.lukicheva@spbu.ru \\ Max Planck Institute for Solar System Research, \\ Gottingen, Germany, m.lukicheva@spbu.ru
}

\begin{abstract}
The paper discusses the diagnostics of plasma jets in the solar corona with the use of data from modern space-born and ground-based telescopes observing the Sun in the extreme ultraviolet (EUV) and microwave bands. We examine observational parameters of EUV and radio emission in events associated with plasma jets, depending on the formation mechanism, initiation conditions, and evolution of the jets. The opportunities provided by the study of plasma jets, which rely on simultaneous observations in different bands, are highlighted. For a number of jets, we have measured their primary parameters; and in this paper we present preliminary results of statistical analysis of the data obtained. Microwave observations of several specific events, made by ground-based instruments RATAN-600, SRH, and Nobeyama Radioheliograph, are considered in detail. The diagnostic capabilities of
\end{abstract}

these instruments for studying coronal jets are shown. To analyze the three-dimensional structure of the coronal magnetic field, we have used SDO/HMI data, which allowed for the reconstruction of the field in the lower corona. The information gained is compared with the results of diagnostics of the magnetic field at the base of the corona according to RATAN-600 data. The purpose of the methods developed is to determine the physical mechanisms responsible for the generation, collimation, and dynamics of plasma jets in the solar atmosphere.

Keywords: Sun, solar corona, solar active regions, coronal jets, microwave observations of the Sun, EUV observations of the Sun.

\section{INTRODUCTION}

Hydrogen plasma of the solar corona (with alpha particles and positive ions of heavier elements) features a high degree of ionization (to $100 \%$ ) and a wide range of temperatures (from $50 \cdot 10^{3}$ to $30 \cdot 10^{6} \mathrm{~K}$ ) and densities $10^{6}-10^{11} \mathrm{~cm}^{-3}$. Observations show that the corona is a very dynamic medium with a wide range of different flows, including those of the jet type. The magnetic field plays a key role (parameter $\beta<0.1$ ) in the formation and dynamics of plasma jets in the solar corona, although there is a possibility that field-aligned plasma flows with velocities of the order of the sound speed are generated by thermodynamic processes. A variety of physical conditions and the possibility of detailed observations make solar plasma a natural laboratory for studying various physical processes responsible for generation, collimation, and propagation of plasma jets. These processes can exhibit both collisional and collisionless modes, thermal and nonthermal particle distributions, and diverse dynamic phenomena (including “cold" and "hot” jets, MHD waves of different types, etc.) [Zhang Ji, 2014; Nisticò et al., 2015; Raouafi et al., 2016; Cho et al., 2020; Yu, Nakariakov, 2020; Joshi et al., 2020].

Continuous monitoring of solar emission in radio, optical, UV, EUV, X-ray, and gamma bands with ground and space-based high-precision instruments provides exciting opportunities for studying and diagnosing plasma jets. Open access to observational data and computer programs of its analysis and simulation enabled us to create a catalog of observations of coronal jets, to continue updating it, and to use it for studying jets.

The paper is organized as follows: Section 1 delves into observational manifestations of coronal jets in EUV and radio bands; Section 2 briefly describes the catalog of coronal jets we have created; Section 3 gives examples of observations of coronal jets in the microwave range; Section 4 and Conclusion discuss findings and the main conclusions.

\section{OBSERVABLE MANIFESTATIONS OF JETS IN DIFFERENT BANDS}

Coronal plasma jets are dynamic plasma structures with a pronounced plasma flow, which are observed in EUV images as relatively short-lived (usually up to several hours) elongated structures. The plasma speed in some events may be as high as $1000 \mathrm{~km} / \mathrm{s}$, which is comparable with the local Alfvén speed. In some cases, the occurrence of a jet is accompanied by a local brightening in 
lower layers and by a change in the surface magnetic field. Sometimes there is a helical fine structure, confirmed by quasi-stereoscopic methods, which indicates the presence of a parallel electric current. As in coronal loops, the density and temperature inside a jet differ from background levels, allowing it to act as a waveguide for MHD waves, including kink oscillations, observed as transverse motions propagating along the jet.

According to coronagraph data, coronal jets occur at different heights in the solar atmosphere, in particular in the upper corona [Kudriavtseva, Prosovetsky, 2019]. We examine the plasma jets in the lower corona, which are clearly seen in SDO/AIA images. Jets observed in the solar corona can be divided into two categories - "hot" and "cold". The former consist of coronal plasma with a temperature of the order of $10^{6} \mathrm{~K}$ and higher, whereas cold jets feature a significant proportion of cold and dense chromospheric material with a temperature of several tens of thousands of kelvin.

Because of the significant difference in temperatures, the observational manifestations of jets of the two types differ too. Hot jets show up as thin and long bright structures seen in SDO/AIA hot EUV channels [Pesnell et al., 2012]. An example of a hot coronal jet image, obtained at a wavelength of $171 \AA$ by SDO/AIA, is given in Figure 1. In a colder $304 \AA$ channel and in IRIS images, hot jets are usually not visible.

Cold jets, in turn, best manifest themselves as bright structures in $304 \AA$ images; a maximum sensitivity of this channel is at $60000 \mathrm{~K}$. In SDO/AIA hotter channels (171, 193, 131, $94 \AA$ ), these structures are also often seen as bunches of dark matter absorbing EUV radiation. In addition to the hot and cold jets there are sometimes jets composed of several components with different temperatures [Zhang, Ji, 2014; Joshi et al., 2020]. Such jets are observed as bright structured objects simultaneously in coronal (e.g., $171 \AA$ ) and chromospheric (304 A) SDO/AIA channels. Such a jet is described in Section 2 .

Thus, in its observable manifestations the material of cold and multicomponent jets often reminds the prominence material, and in some cases it becomes difficult to distinguish these jets from surges and eruptions of small filaments. A cold and dense fraction of such jets when entering the corona can shield an underlying cyclotron microwave source and cause a short-term decrease in its apparent brightness. This phenomenon has been identi-

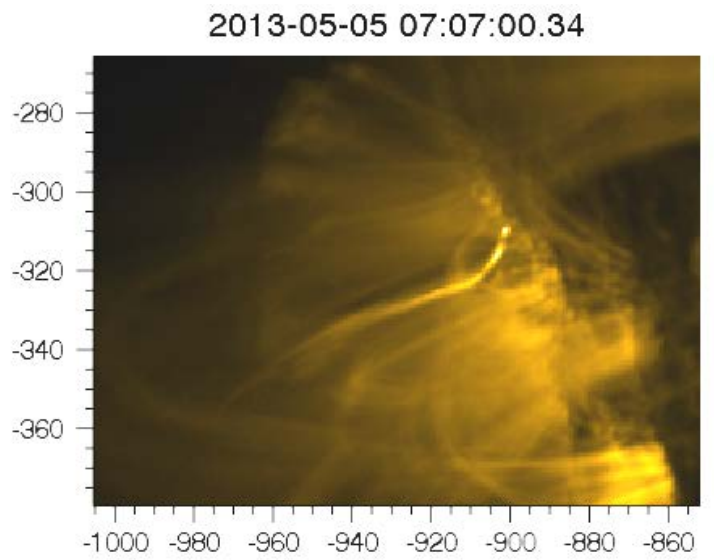

fied from the imaging data acquired by the Nobeyama Radioheliograph (NoRH) [Nakajima et al., 1994] and the Siberian Solar Radioheliograph (SRH) [Lesovoi et al., 2017] and is now known as negative microwave bursts [Kuzmenko et al., 2009; Kuzmenko, 2020].

Also of particular interest for diagnostics of coronal jets is the process of their initiation. According to conventional concepts, a jet is driven by plasma acceleration in its footpoint through magnetic reconnection, which may occur, in particular, during microflares. Since characteristics of a microflare largely determine parameters of the induced jet, observations and analysis of short EUV, radio or X-ray bursts during initiation of jets provide important information for their diagnostics.

\section{CATALOG OF CORONAL JETS}

To diagnose coronal jets and study statistical scaling of their parameters, we have created a jet observation catalog [Kaltman et al., 2019], available on the website [http://spbf.sao.ru/coronal-jets-catalog]. The catalog is based on data on coronal jets from the Heliophysics Events Knowledgebase (HEK) and includes observations obtained with the space-borne high-precision EUV imaging telescope SDO/AIA, as well as with groundbased telescopes and spectrometers, including the Radio Telescope of the Academy of Sciences (RATAN-600) [Bogod, 2011], SRH, and NoRH. In addition, the catalog contains radio maps and light curves from NoRH and SRH for those cases where a jet or the process of its initiation was observed by the aforementioned telescopes.

The catalog also includes contextual observations such as microwave images of active regions with jets at the frequency of $17 \mathrm{GHz}(\mathrm{NoRH})$ and at several frequencies in the $4-8 \mathrm{GHz}$ band (SRH), as well as RATAN-600 high-precision measurements of microwave radiation spectra. The last ones allow us to estimate the magnetic field at the base of the corona and supplement data on coronal magnetic field reconstruction in the nonlinear force-free field (NLFFF) approximation, which is also presented in the catalog (for more detail see [Kaltman et al., 2019; Stupishin et al., 2020]).

For preprocessing of available data, we have developed an algorithm for automatic detection of jets in EUV

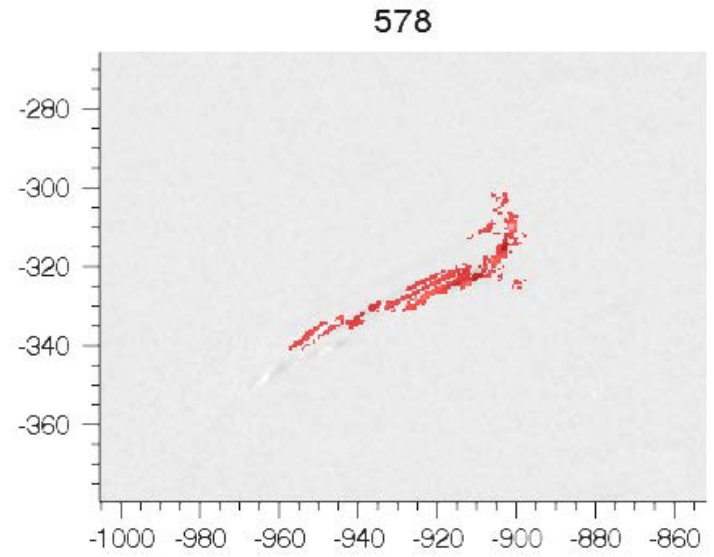

Figure 1. Example of automatic identification of a coronal jet in SDO/AIA images in the $171 \AA$ channel 
image series [Stupishin et al., 2020]. The program that implements the algorithm automatically identifies one or more jet components for each event in the catalog and flags them in images and video files (see Figure 1). Furthermore, we measure and store in the catalog the following parameters of the details identified:

- Tstart is the jet onset time;

- Tmax is the time of jet maximum;

- Tend is the jet end time;

- Duration is the jet lifetime;

- Max cardinality is the maximum number of pixels;

- Max aspect ratio is the maximum elongation;

- Min aspect ratio is the minimum elongation;

- $X$ from, $X$ to, $Y$ from, $Y$ to are coordinates of the bounding rectangle (for the entire jet history).

The parameters found by the algorithm may be used for further statistical analysis of jet characteristics. For example, for preliminary sampling of 80 events we have esti- mated some parameters of the jets found (in brackets are mean values): the duration is from 1 to $17 \mathrm{~min}$, the maximum/minimum aspect ratio is respectively 4-21 (7.3) / 3.5-7.5 (3.9 ) arcsec., and the length is 13-144 (97) arcsec.

Since the algorithm runs independently in different SDO/AIA channels, we can perform a comparative analysis of visibility of individual components of jets at different wavelengths corresponding to different emitting plasma temperatures. The table gives examples of paired observations of jets in the 171 and $193 \AA$ lines. The pairs have been selected according to the coincidence of the jet maximum and duration (Tmax and Duration columns), and also according to its location ( $X$ from, $X$ to, $Y$ from, $Y$ to). Independent analysis shows the following tendency in different channels: jets at $193 \AA$ have, on average, shorter length and duration than at $171 \AA$.

Results of the comparative analysis of jet components visible in the 171 and $193 \AA$ channels

\begin{tabular}{|c|c|c|c|c|c|c|c|c|c|c|c|}
\hline T start & $T \max$ & Duration & Max card. & Max as.rat. & Min as.rat. & $x$ from & $x$ to & Y from & $\mathrm{Y}$ to & Length & Line, $\mathrm{A}$ \\
\hline 2016-04-28T09:58:05.84 & 2016-04-28T09:58:05.84 & $66^{\prime} 12$ & 143 & 9,36 & 3,53 & 471,2 & 496,9 & 14,2 & 23,8 & 27,43447 & 193 \\
\hline 2017-09-04T03:49:40.84 & 2017-09-04T03:50:40.84 & $2^{\prime} 12$ & 276 & 10,04 & 3,73 & $-841,8$ & -820 & 244,7 & 275,9 & 38,29204 & 193 \\
\hline 2017-09-04T03:49:45.34 & 2017-09-04T03:50:45.34 & $2^{\prime} 12$ & 291 & 7,65 & 3,63 & -846 & -820 & 245,3 & 280,1 & 43,68066 & 171 \\
\hline 2017-09-04T04:19:16.84 & 2017-09-04T04:19:52.84 & $2^{\prime} 12$ & 124 & 5,58 & 3,87 & -814 & -796 & 246,6 & 275,4 & 33,96233 & 193 \\
\hline 2017-09-04T04:19:21.35 & 2017-09-04T04:20:09.35 & $2^{\prime} 24$ & 139 & 5,56 & 3,51 & $-812,8$ & -794 & 248,4 & 276,6 & 34,17206 & 171 \\
\hline 2017-09-13T05:51:40.84 & 2017-09-13Т05:52:16.84 & $1^{\prime} 24$ & 140 & 7,08 & 4,52 & $-531,6$ & -508 & $-3,4$ & 26,6 & 38,29412 & 193 \\
\hline 2017-09-13T05:51:45.35 & 2017-09-13T05:53:45.35 & 3'12 & 175 & 7,63 & 3,58 & $-530,6$ & -508 & $-2,2$ & 24,2 & 34,68732 & 171 \\
\hline 2018-04-03T04:04:28.84 & 2018-04-03T04:05:28.84 & $1^{\prime} 12$ & 1113 & 5,11 & 3,89 & 338,2 & 380,7 & 3,4 & 106,7 & 111,7011 & 193 \\
\hline 2018-04-03T04:04:33.35 & 2018-04-03T04:07:09.35 & $2^{\prime} 48$ & 1708 & 5,29 & 3,81 & 336,2 & 393,8 & $-12,4$ & 139,3 & 162,2672 & 171 \\
\hline 2018-09-14T05:48:40.84 & 2018-09-14T05:50:52.84 & 3'12 & 147 & 6,1 & 3,62 & 189,5 & 220,9 & $-287,1$ & $-273,9$ & 34,06171 & 193 \\
\hline 2018-09-14T05:48:21.35 & 2018-09-14T05:51:09.35 & $4^{\prime} 12$ & 179 & 6,7 & 3,62 & 188 & 220,3 & $-288,9$ & $-274,5$ & 35,36453 & 171 \\
\hline 2018-10-13T05:09:52.85 & 2018-10-13Т05:10:04.85 & $1^{\prime} 24$ & 526 & 4,66 & 3,71 & $-812,5$ & -763 & $-165,6$ & $-146,4$ & 53,37303 & 193 \\
\hline 2018-10-13T05:09:21.35 & 2018-10-13T05:11:09.35 & $2^{\prime} 24$ & 539 & 5,37 & 3,59 & $-816,1$ & -763 & $-166,8$ & -147 & 56,48407 & 171 \\
\hline
\end{tabular}

\section{OBSERVATION OF JETS IN A MICROWAVE RANGE}

In terms of sensitivity, spectral resolution, and width of the spectral band, RATAN-600 has the best characteristics among existing radio telescopes able to regularly observe the Sun. However, due to limitations related to the scanning mode, RATAN-600 observations are generally used only for gathering contextual information about an active region in which a jet is detected. Nonetheless, under favorable conditions, we can observe the radio brightness variations directly linked to the jet. For one of the events from our catalog, the development of a jet coincided in time with several RATAN-600 scans. Figure 2 presents a RATAN-600 scan on the background of active region images in different SDO/AIA/HMI channels; Figure 3 shows several successive scans, some of which were made during occurrence of a jet. Clearly noticeable is an increase in radio source polarization (Stokes V) when the jet was within the RATAN-600 beam.
The radioheliographs NoRH and SRH, which record a full two-dimensional radio map of the entire solar disk with a cadence of $\sim 10 \mathrm{~s}$ for each frequency, provide somewhat greater opportunities for direct observation of coronal jets.

An example of simultaneous observation of a jet with NoRH and SRH in the radio band is the event recorded in AR 12680 on September 13, 2017.

The jet from AR 12680 is classified as multicomponent, and its material contains fractions of both hot coronal plasma and colder and denser chromospheric material. In EUV images, this jet is seen in several SDO/AIA channels, in particular in the $304 \AA$ channel, as a bright elongated structure. In the radio band, this jet was observed simultaneously by NoRH and SRH. Figure 4 shows a radio map of the microwave radiation intensity $I$ at the frequency of $17 \mathrm{GHz}$ (NoRH), superimposed on an EUV image in the $171 \AA$ line. There is a high correlation between the shape and size of the jet, identified in the $171 \AA$ AIA images at different times, and changes in the shape of the radio source at the frequency of $17 \mathrm{GHz}$. This demonstrates that 

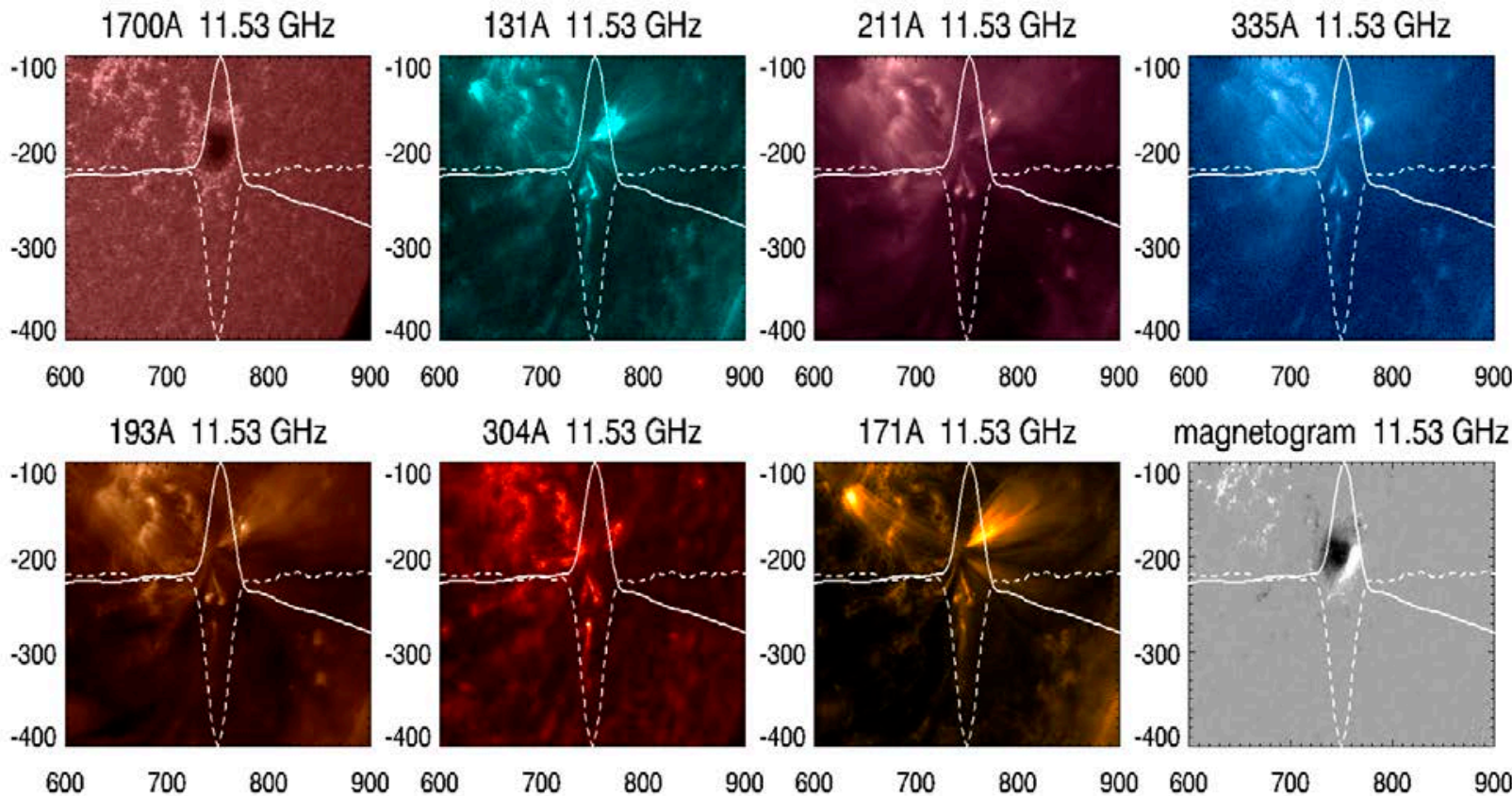

Figure 2. SDO (AIA and HMI) images captured on April 17, 2019 at 08:32 UT (AIA) and 08:33 UT (HMI) with superimposed scans in antenna temperatures (solid line — intensity $I$, dashed line — polarization V), obtained by RATAN-600 at 08:32 UT at a frequency of $11.53 \mathrm{GHz}$ during occurrence of a jet (08:28-08:47 UT). The jet is located vertically within the scan $V$. When overlapped, the images are rotated so that the RATAN-600 beam in the image is directed vertically
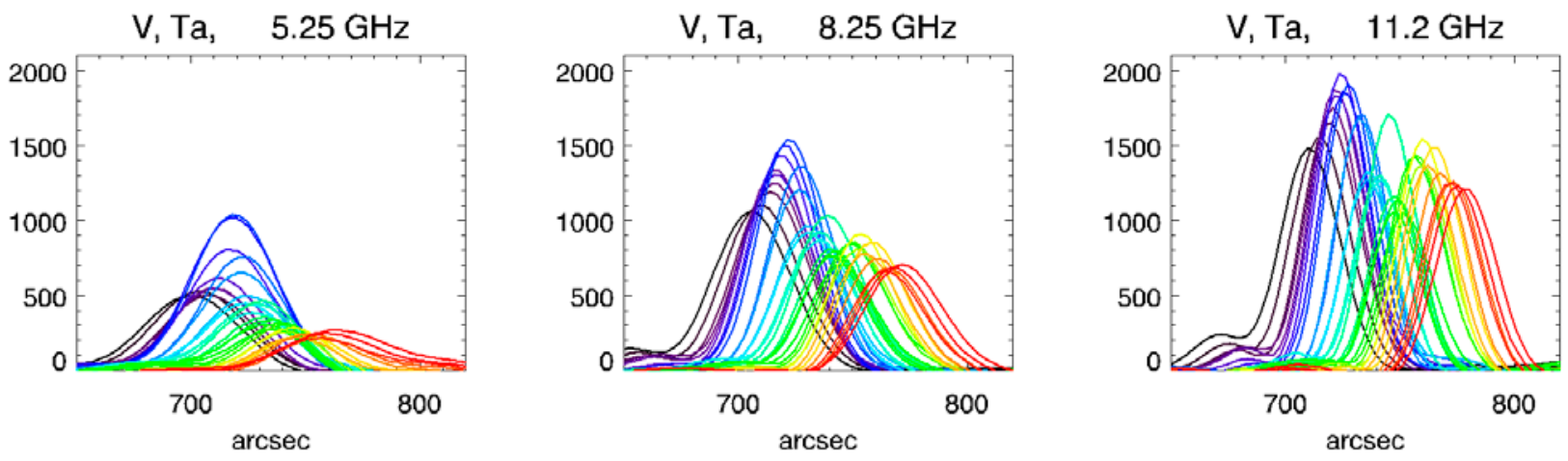

Figure 3. RATAN-600 scans of antenna temperatures of polarized radiation in AR 12738 on April 17, 2019 at frequencies of 5.25, 8.25, and 11.20 GHz. Different colors indicate observations in different azimuths: 31 records in a time span 07:11-11:15 UT with a step of $8 \mathrm{~min}$. The left-to-right shift of the scans is a projection effect associated with the Earth rotation and depending on the orientation of a radio telescope antenna. Jet passage time corresponds to a radiation increase in blue scans

in this case the jet is observed directly at the frequency of $17 \mathrm{GHz}$.

In addition to $17 \mathrm{GHz}$, this event was also recorded by SRH in 4-8 GHz. Although the limited spatial resolution did not allow us to capture a direct image of the jet, we detected an increase in the radio flux from the active region.

Figure 5 presents radio maps of the full solar disk and light curves of the microwave flux from the active region in which the jet was observed. Radio response is a superposition of the long-lived increase in the brightness that seems to be directly linked to the jet and the short strongly polarized radio burst that was observed during initiation of the jet. The latter is likely to be associated with the microflare that excited the jet.
Although the time of jet occurrence was not covered by daily solar observations performed by RATAN-600, its data was used to gain additional information about maximum magnetic field strength in the upper part of the transition zone/base of the corona, where the footpoint of the jet might have been located. Figure 6 shows the microwave radiation spectrum for AR 12680 , obtained from RATAN-600 observations. While the observations were made four hours after the event, they can be used to obtain contextual information about the coronal magnetic field because there were no significant changes in configuration of AR or solar flares in it during this period.

From the polarized radiation spectrum, we have found out that the maximum frequency of cyclotron radiation is in the range $13.3-13.4 \mathrm{GHz}$. From these frequencies, 

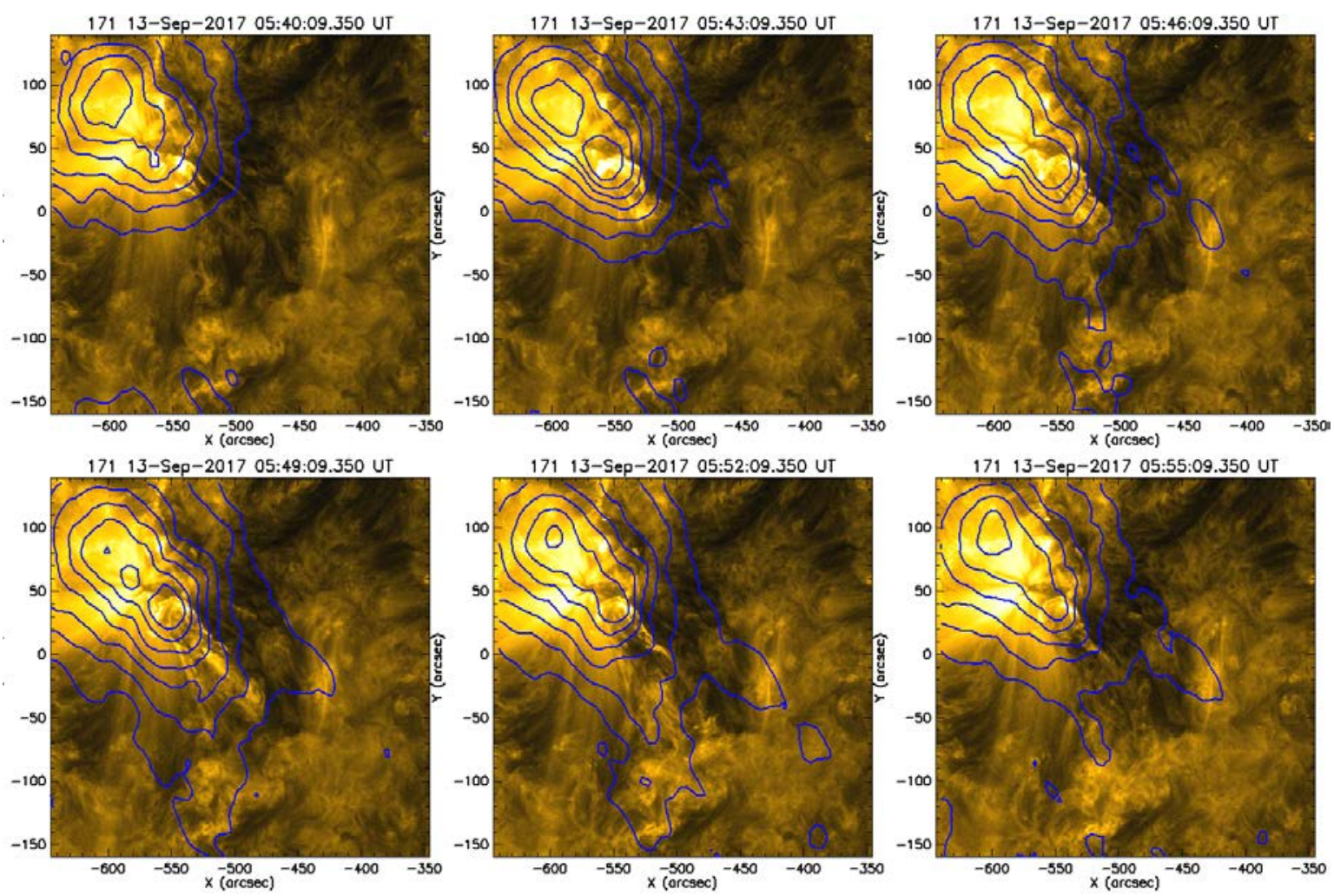

Figure 4. Radio maps of microwave radiation at a frequency of $17 \mathrm{GHz}$ obtained by NoRH on September 13, 2017 for different stages of jet development and superimposed on SDO/AIA $171 \AA$ images as contours. The radio brightness contours correspond to values from 10500 to $15000 \mathrm{~K}$ with an increment of $500 \mathrm{~K}$

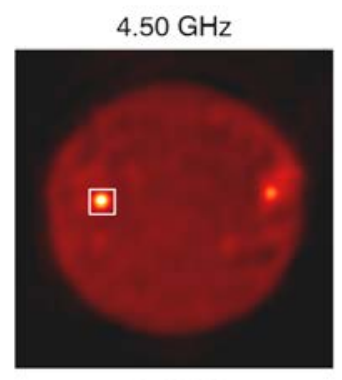

$6.00 \mathrm{GHz}$

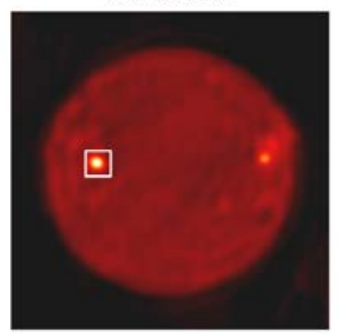

$7.50 \mathrm{GHz}$

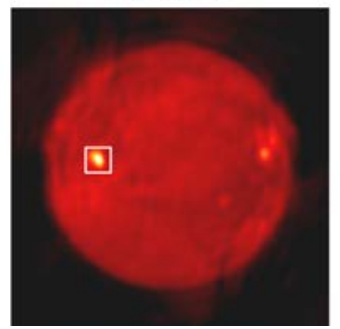

$5.20 \mathrm{GHz}$

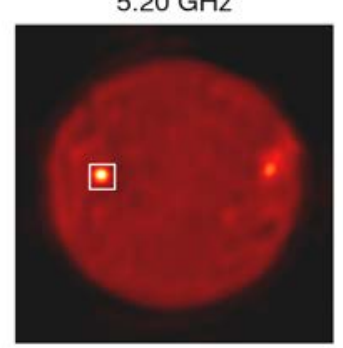

$6.80 \mathrm{GHz}$

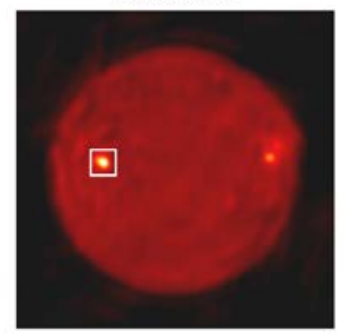

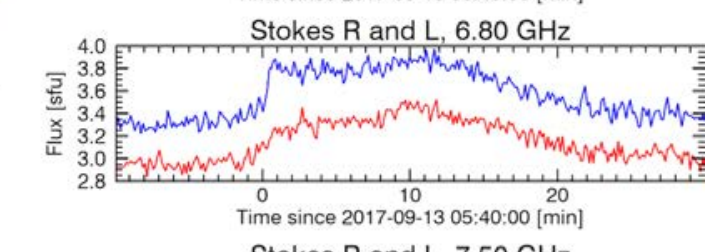

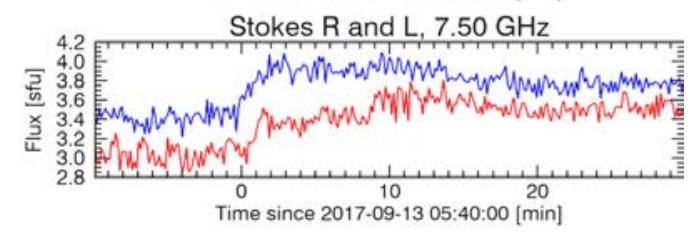

Figure 5. Radio images of the Sun (left) from SRH for September 13, 2017. The active region in which the jet was observed is marked with a white rectangle. Microwave flux light curves for this region in the right (RCP, blue) and left (LCP, red) polarization are shown in the right panels 


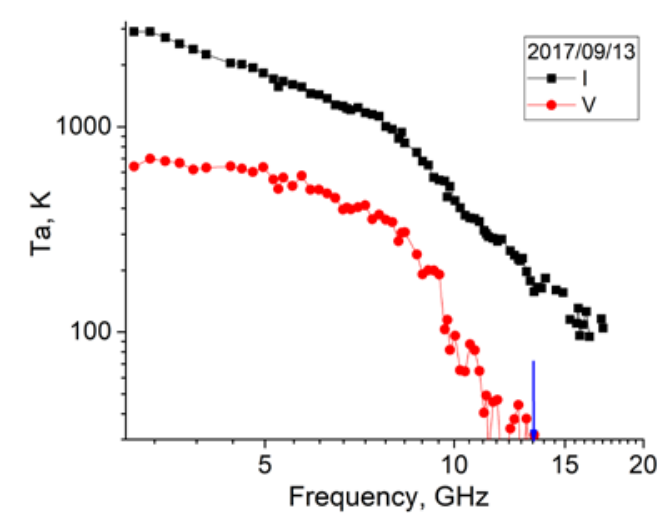

Figure 6. Spectrum of antenna temperatures ( $I$ - intensity; $V$ - polarization) of AR 12680 for September 13, 2017, as derived from RATAN-600 observations (09:09:30 UT). The blue arrow indicates the maximum frequency of cyclotron radiation

we have estimated the magnetic field at 1580-1620 G in the upper layers of the transition zone on the axis of the radio source over a sunspot in the assumption that emission in the extraordinary mode occurs in the third harmonic of gyrofrequency.

To verify the magnetic field measurements from microwave spectra and to study its morphology, we have reconstructed the coronal magnetic field in the NLFFF approximation. Comparison of the magnetic field measurements at the base of the corona, carried out by analyzing the microwave spectrum (1580-1620 G), with magnetic field reconstruction data has shown that the value obtained corresponds to a height of $\sim 1600 \mathrm{~km}$ above the sunspot umbra and is consistent with the expected heights of the transition layer between the chromosphere and the corona in the atmosphere of sunspots. Furthermore, we analyzed the magnetic field line structure in the region where the jet occurred. Comparison with jet images in the $171 \AA$ channel reveals that field lines of the reconstructed magnetic field match well with the jet shape. The reconstructed magnetic field structure and its comparison with an EUV jet image are shown in Figure 7.

\section{DISCUSSION}

According to current views, the jet phenomena observed in the solar corona are complex processes including impulsive and potentially quasistationary energy releases through magnetic reconnection, as well as plasma heating and charged particle acceleration. In terms of magnetic reconnection, jets are an example of the distribution of released energy alternative to flares. In jets, the main part of magnetic energy is converted not into particle acceleration and plasma heating, but into kinetic energy of macroscopic plasma flows. At the same time, the ratio of the energy fractions responsible for particle acceleration and plasma heating remains unknown and needs to be estimated. Moreover, an interesting question is whether the jet results from the acceleration localized in time or its acceleration continues throughout its evolution. Of particular interest are the jet collimation mechanisms that prevent its rapid expansion, transverse filamentation, various instabilities (specifically, Kelvin-Helmholtz and negative energy waves), and turbulence.

Answering these questions requires us to analyze observations in different bands providing information both on the plasma temperature and density in the jet and on accelerated particles. Such observations may also open up an opportunity to determine, at least approximately, the magnetic configuration of both the active region in which the jet is initiated and the background plasma with which the jet interacts. This condition explains the need to combine observations not only at different wavelengths in the EUV band but also in a broad spectral band in one catalog. Yet there is a need for information about photospheric distribution of sources of the coronal magnetic field for its reconstruction by extrapolation.
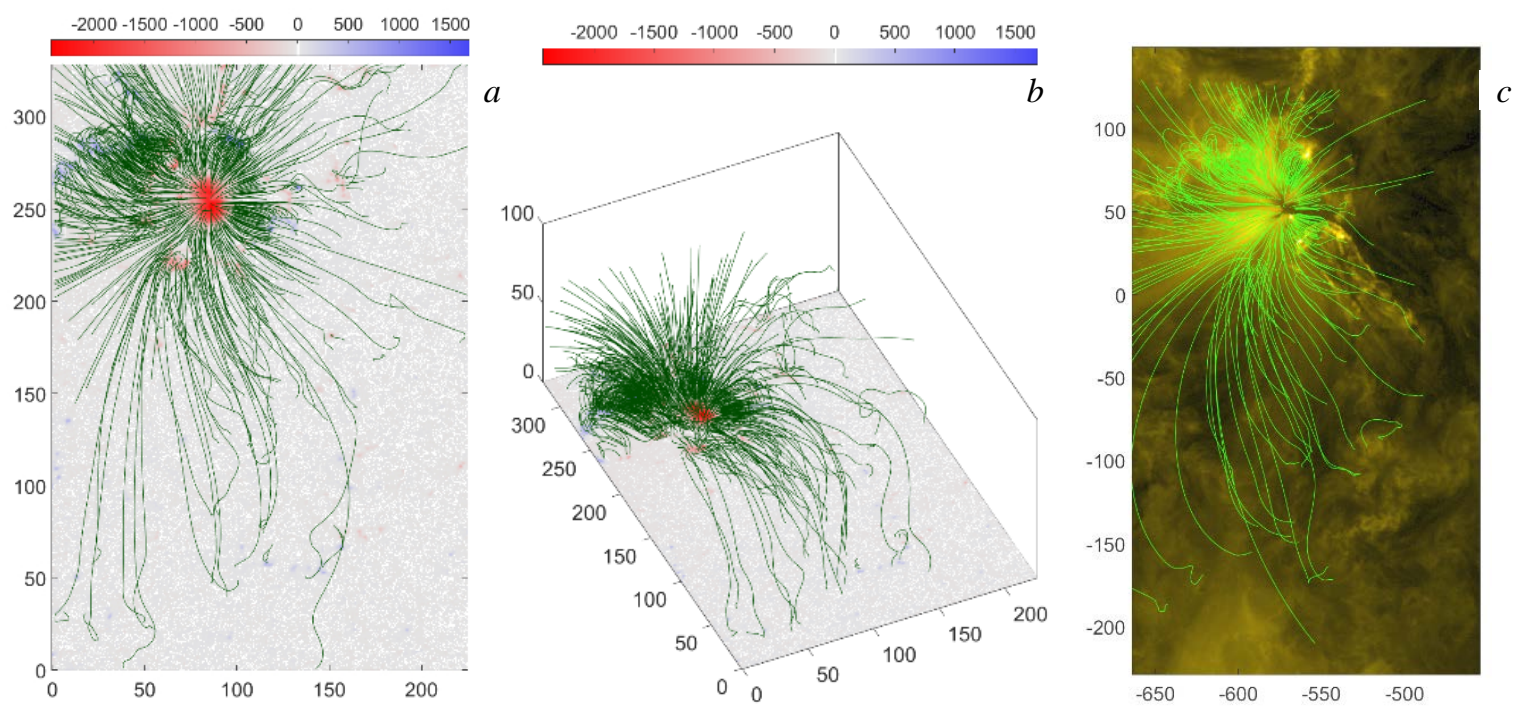

Figure 7. Magnetic field lines in AR 12680 according to NLFFF extrapolation data, superimposed on a magnetogram: top view (a), side view $(b)$, and view from Earth against an EUV image in the $171 \AA$ SDO/AIA channel (c) 
Of particular interest is to identify experimental correlations of observable jet parameters (lifetime, velocity, length, and width) between themselves and with computed parameters such as Alfvén, sound, and tube speeds. This information is crucial for the assessment of jet energy and power, which is necessary to reveal the mechanism for its generation. In this context, it is also highly interesting to estimate the energy and spectrum of nonthermal electrons accelerated during the energy release. In the case of jet generation during magnetic reconnection, this allows us to determine the distribution of the converted magnetic field energy from the released energy of three main types (direct plasma heating, acceleration of charged particles, and kinetic energy of jets), and the possible evolution of this distribution at different stages of the process.

Another important task is to classify the jets by several types, according to correlations of different observable parameters such as velocities and their evolution (acceleration, deceleration), temperatures, morphology, characteristics of accompanying radio emission, etc. This allows us to use the theoretical modeling results for determining which acceleration and collimation mechanisms work in jets of a particular type. Different theoretical models can also be tested by comparing theoretically predicted correlations of different parameters with observed ones. A similar technique has recently been successfully applied to the analysis of the mechanisms responsible for the excitation of kink oscillations of coronal loops, which is based on a special catalog of corresponding events [Zimovets et al., 2015]. A separate task is to determine inclination angles of jet axes with respect to the solar surface and their evolution during a solar cycle, which will significantly improve the reconstruction of the global geometry of the coronal magnetic field by the method proposed in [Fleishman et al., 2017].

The catalog we have developed is of particular interest for identifying the relationships of wave process observed in jets (specifically, kink and slow oscillations) with jet parameters, as well as for their seismological diagnostics. In particular, a possibility of estimating flow velocity variations by slow magnetoacoustic waves has recently been established and utilized [Cho et al., 2020]. In the case of kink waves, which relate to quick branches of magnetohydrodynamic waves, it is interesting to study their roles in initiating the effects associated with waves of negative energy (see, e.g., [Yu, Nakariakov, 2020]), which may significantly change our understanding of MHD-wave generation in the corona and solar wind.

\section{CONCLUSION}

The paper deals with observable manifestations of coronal jets in different bands of the electromagnetic spectrum. Using several events as an example, we have shown the potential for diagnostics of jets by analyzing SDO/AIA, RATAN-600, SRH, and NoRH observations. The jets were studied using images in EUV and radio bands and microwave spectra obtained with RATAN600 . The data was supplemented with information on configuration of the coronal magnetic field reconstructed in the NLFFF approximation from SDO/HMI photospheric magnetograms.

Using the multicomponent coronal jet that occurred in AR 12680 on September 13, 2017 as an example, we have demonstrated an approach to plasma jet diagnostics based on observations in microwave and EUV bands. From NoRH data we have obtained a series of jet images at a frequency of $17 \mathrm{GHz}$, which spatially coincide with the EUV image of a jet observed at 171 $\AA$. Moreover, this event was observed by SRH at five frequencies in the $4-8 \mathrm{GHz}$ band as an increase in the microwave flux from the parent active region throughout the lifetime of the jet and the short strongly polarized radio burst during its initiation. The magnetic field at the base of the corona in this active region was estimated from RATAN-600 microwave spectra as 15801620 G. The coronal magnetic field configuration was reconstructed in the NLFFF approximation from SDO/HMI photospheric magnetograms. Field lines of the reconstructed magnetic field in the projection on the plane of the sky match well with the visible contours of the jet and can therefore be used to assess its geometric characteristics such as height and inclination angle.

The presented approach to the analysis of plasma jets observed simultaneously in different bands allows in-depth study of a wide variety of jet flows occurring in the solar atmosphere at all phases of the solar cycle, including their initiation, energy, dynamics, and collimation, as well as related plasma processes: instability, turbulence, and acceleration of charged particles [Raouafi et al., 2016; Cho et al., 2020; Yu, Nakariakov, 2020; Zhang, Ji, 2014; Joshi et al., 2020].

The work was financially supported by RFBR (Grant No. 18-29-21016) and by the Ministry of Science and Higher Education of the Russian Federation (Government Assignments from SAO RAS, ISTP SB RAS, and $\mathrm{SPbU}$ ). The results were obtained using the equipment of Shared Equipment Center «Angara» [https://ckp-rf.ru/ckp/3056].

\section{REFERENCES}

Bogod V.M. RATAN-600 radio telescope in the $24^{\text {th }}$ solar activity cycle. I. New opportunities and tasks. Astrophys. Bull. 2011, vol. 66, no. 2, pp. 190-204. DOI: 10.1134/S199034 1311020064.

Cho I., Nakariakov V.M., Moon Y., Lee J., Yu D., Cho K., Yurchyshyn V., Lee H. Accelerating and supersonic density fluctuations in coronal hole plumes: signature of nascent solar winds. Astrophys. J. 2020, vol. 900, no. 2, p. L19. DOI: 10.3847/2041-8213/abb020.

Fleishman G.D., Anfinogentov S., Loukitcheva M., Mysh'yakov I., Stupishin A. Casting the coronal magnetic field reconstruction tools in 3D using the MHD Bifrost model. Astrophys. J. 2017, vol. 839, no. 1, p. 30. DOI: 10.3847/15384357/aa6840.

Joshi R., Chandra R., Schmieder B., Moreno-Insertis F., Aulanier G., Nóbrega-Siverio D., Devi P. Case study of multitemperature coronal jets for emerging flux MHD models. Astron. Astrophys. 2020, vol. 639, p. A22. DOI: 10.1051/00046361/202037806. 
Kaltman T.I., Nakariakov V.M., Anfinogentov S.A., Stupishin A.G., Loukitcheva M.V., Shendrik A.V. Catalogue of hot jets in the solar corona for 2015-2018. Proc. the XXIII All-Russian Annual Conference "Solar and Solar-Terrestrial Physics”. St. Petersburg, 2019, pp. 197-200. DOI: 10.31725/0552-5829-2019-197-200. (In Russian).

Kudriavtseva A.V., Prosovetsky D.V. White-light polar jets on rising phase of solar cycle 24. J. Atmos. Solar-Terr. Phys. 2019, vol. 193, p. 105039. DOI: 10.1016/j.jastp.2019.05.003.

Kuz'menko I.V., Grechnev V.V., Uralov A.M. A study of eruptive solar events with negative radio bursts. Astron. Reports. 2009, vol. 53, no. 11, pp. 1039-1049. DOI: 10.1134/ S1063772909110092.

Kuzmenko I.V. Coronal jets as a cause of microwave negative bursts. Solar-Terr. Phys. 2020, vol. 6, no 3, pp. 23-28. DOI: $10.12737 /$ stp-63202003.

Lesovoi S., Altyntsev A., Kochanov A., Grechnev V., Gubin A., Zhdanov D., et al. Siberian Radioheliograph: First results. Solar-Terr. Phys. 2017, vol. 3, no. 1, pp. 3-18. DOI: 10.12737/article_58f96ec60fec52.86165286.

Nakajima H., Nishio M., Enome S., Shibasaki K.; Takano T., Hanaoka Y., et al. The Nobeyama Radioheliograph. Proc. IEEE. 1994, vol. 82, no. 5, p. 705-713.

Nisticò G., Zimbardo G., Patsourakos S., Bothmer V., Nakariakov V.M. North-south asymmetry in the magnetic deflection of polar coronal hole jets. Astron. Astrophys. 2015, vol. 583. p. A127. DOI: 10.1051/00046361/201525731.

Pesnell W.D., Thompson B.J., Chamberlin P.C. The Solar Dynamics Observatory (SDO). Solar Phys. 2012, vol. 275, no. 1-2, pp. 3-15. DOI: 10.1007/s11207-011-9841-3.
Raouafi N.E., Patsourakos S., Pariat E., Young P.R., Sterling A.C., Savcheva A., et al. Solar coronal jets: observations, theory, and modeling. Space Sci. Rev. 2016, vol. 201, no. 1-4, pp. 1-53. DOI: 10.1007/s11214-016-0260-5.

Stupishin A.G., Kaltman T.I., Anfinogentov S.A. On the method for selecting jets in homogeneous time series of images of the Sun. Proc. the XXIV All-Russian Annual Conference "Solar and Solar-Terrestrial Physics". St. Petersburg, 2020, pp. 285-288. DOI: 10.31725/0552-5829-2020-285-288. (In Russian).

Yu D.J., Nakariakov V.M. Excitation of negative energy surface magnetohydrodynamic waves in an incompressible cylindrical plasma. Astrophys. J. 2020, vol. 896, no. 1, p. 21. DOI: 10.3847/1538-4357/ab8d3c.

Zhang Q.M., Ji H.S. Blobs in recurring extreme-ultraviolet jets. Astron. Astrophys. 2014, vol. 567, p. A11. DOI: 10.1051/ 0004-6361/201423698.

Zimovets I. V., Nakariakov V.M. Excitation of kink oscillations of coronal loops: statistical study. Astron. Astrophys. 2015, vol. 577, p. A4. DOI: 10.1051/0004-6361/201424960.

URL: http://spbf.sao.ru/coronal-jets-catalog (accessed March 9, 2021).

URL: https://ckp-rf.ru/ckp/3056 (accessed March 9, 2021).

This paper is based on material presented at the 16th Annual Conference on Plasma Physics in the Solar System, February $8-12$, 2021, IKI RAS.

How to cite this article

Anfinogentov S.A., Kaltman T.I., Stupishin A.G., Nakariakov V.M., Loukicheva M.A. Diagnostics of plasma jets in the solar corona. Solar-Terrestrial Physics. 2021. Vol. 7. Iss. 2. P. 3-10. DOI: 10.12737/stp72202101. 